\title{
Iterative building optimization via sequential local optimization operations on distinct attribute clusters of design variants
}

\author{
Ardeshir Mahdavi, Hamidreza Shirdel \\ Department of Building Physics and Building Ecology \\ TU Wien, Vienna Austria
}

\begin{abstract}
This paper presents the current state of development concerning an iterative approach to simulation-assisted building performance optimization. Thereby, clusters of design space attributes (i.e., meaningful subsets of design parameters) are subjected to multiple passes of local simulation-assisted optimization runs. Hence, instead of allocating an individual dimension to each and every variable of a complex design within the context of a single-pass global optimization campaign, multiple iterative optimization steps target coherent clusters of such attributes and pursue those until further performance improvement is not forthcoming. The paper describes a number of application cases of the proposed approach.
\end{abstract}

\section{Introduction and background}

This paper presents the current state of development concerning an iterative approach to simulation-assisted building performance optimization. The research study explores the potential of a novel path toward iterative global optimization of locally optimized attribute clusters of building design solutions (Mahdavi et al. 2016). The pursuit of this specific approach was motivated by a number of observations pertaining to conventional optimization practices and strategies in building design and operation.

The application of numerical optimization in the design process is not a recent phenomenon. In fact, research and development efforts in this domain have a track record over multiple decades, resulting in advanced in building informatics and mathematical optimization methods (Nguyen et al. 2014). Managing a large number of independent variables in the optimization process still represents a challenge (Wetter, 2016). Frequently, stochastic optimization algorithms, such as GA (genetic algorithm) and PSO (Particle Swarm Optimization), are deployed to solve optimization problems pertaining to building simulation models (Yang, et al., 2014). The combined application of building simulation applications (Fiske \& Bhonde, 2015) and optimization tools have been suggested to support the design of high performance buildings are more likely to result from an effective performance-based design process.

In the past, multiple developments have led to the realization of global optimization routines that can be applied to large multi-dimensional design option spaces. Such developments have benefited from the increasing power of computational devices, enhancement of optimization algorithms, and progress in construction of computationally efficient surrogate models in lieu of explicit simulation procedures. These developments have been highly fruitful, but they also entail certain drawbacks. For one thing, surrogate models do not always capture the behaviour of designs reliably, especially those with utterly complex or non-conventional features. A second problem is related to the potentially opaque nature of large-scale global optimization exercises. As such, it is not necessarily obvious to the user of a global optimization tool, how the complex logic of multiple interrelated design attributes and their specific values are handled toward to recommended solution, which is then to be taken at face value (Shirdel et al. 2018). This circumstance makes (especially a one-shot) global optimization concept less amenable to the provision of intuitively comprehensible support in typical design processes, which are rather iterative in nature.

There may be different ways of avoiding the extremes of a one-shot global optimization on the one hand and the tedious one variable at a time parametric study on the other hand. In the specific approach we have been developing, clusters of design space attributes (i.e., meaningful subsets of design parameters) are subjected to multiple passes of local simulation-assisted optimization runs. Hence, instead of allocating an individual dimension to each and every variable of a complex design within the context of a single-pass global optimization campaign, multiple iterative optimization steps target coherent clusters of such attributes and pursue those until the overall design meets the expected performance (or until further performance improvement is not forthcoming).

Note that there is not a unique receipt for the formation of design attribute clusters. As a result, those proposed and implemented in our research serve mainly illustrative purposes. The objective is to provide potential users with the possibility and flexibility to define and modify clustering schemes. The paper describes a number of application cases of the proposed approach. Thereby, the method's advantages are demonstrated, namely the use of original simulation models instead of meta-models, as well as provision of support for the iterative, transparent, and intuitive navigation of the design space. The ideas presented in this paper have thus the potential to further advance the integration of coupled simulation and optimization environments in the building process (Mahdavi et al. 2016). 


\section{The iterative cluster-based optimization approach}

\section{About clusters}

Clusters are meant here to denote groupings of design attributes that are intuitively comprehensible to typical building designers as a compound yet coherent aspects of a design. Examples of such cluster could include building geometry variables, material properties, building construction attributes, features of technical devices, renewable energy elements, building control parameter, and many more. As there is not a single valid grouping scheme for such variables, the present implementation targets scalability and flexibility. Specifically, users are to be provided with degrees of freedom in view of the selection of the clusters to be optimized and the composition of each cluster. Moreover, additional clusters can be defined and variable sets in each cluster can be manipulated, while still achieving convergence within reasonable temporal horizons. Iterative optimization run can follow different patterns, including predefined sequences (Figure 1) or random cycling between attribute clusters (Figure 2).

\section{Variables and performance indicators}

Simulation-powered optimization supported building design must address the multiple potentially influential design and context variables on targeted performance criteria (e.g., energy use). The main categories of salient variables relate to design control. Design variables cover geometry attributes as well as the material and construction properties such as those relevant to building envelope. The control variables are relevant to the operation of building systems (e.g., dynamic elements of the building enclosure, such as blinds, windows, and luminaires).

Amongst the multitude of relevant performance indicators, the current contribution focuses on buildings' energy demand for heating, cooling, and lighting. The performance indicators are the annual heating, cooling and electric light energy demand of the building. Needless to say, depending on the location of the building and priorities of the designers, various formulations of objective functions can be conceived and used to guide the convergence process toward optimized design solutions.

In the next two illustrative examples, different variables are organized into cluster sets and made subject to an iterative process of energy performance optimization.

\section{The first illustrative case study}

\section{Building and energy model}

The base model for the first case study is a modified version of a standard ASHRAE small office building retrieved from Commercial Prototype Building Models supported by the U.S. Department of Energy (DOE 2016). The office's dimensions (length, width, and height) are approximately $27.7,18.5$, and $3 \mathrm{~m}$ and it is assumed to be located in Vienna, Austria. Five thermal zones (one core zone and four perimeter zones) are defined (Figure 3).

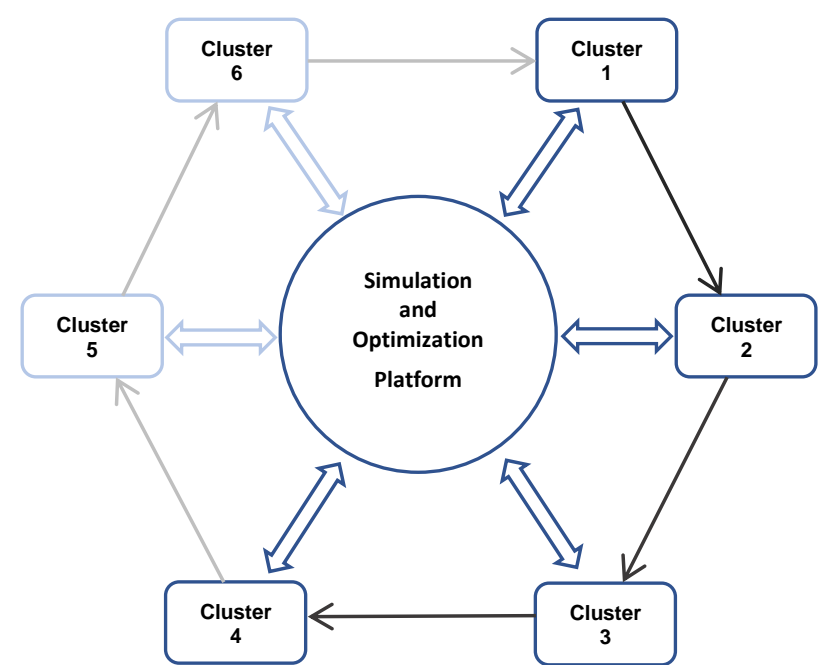

Figure 1: Illustration of predefined iteration between the attribute clusters.

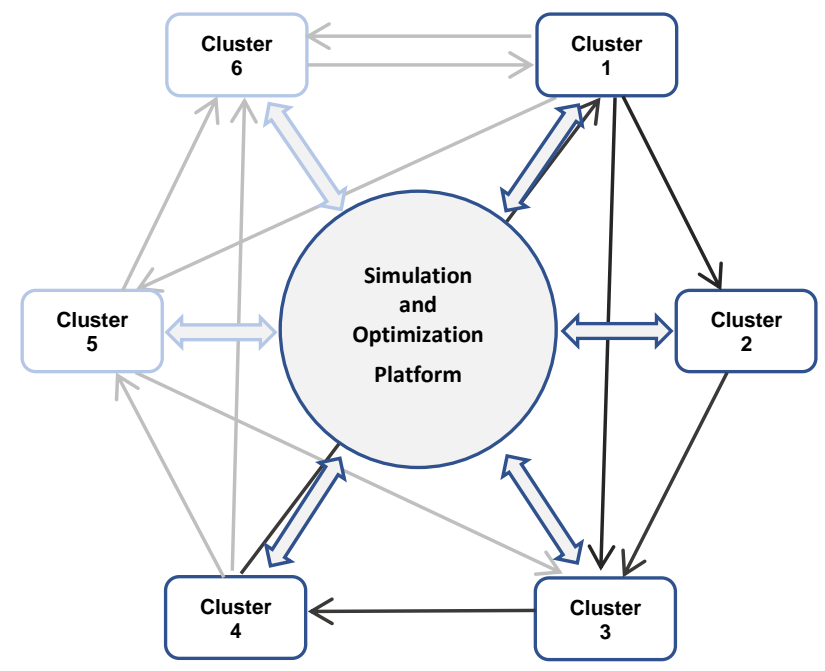

Figure 2: Illustration of random cycling between the attribute clusters.

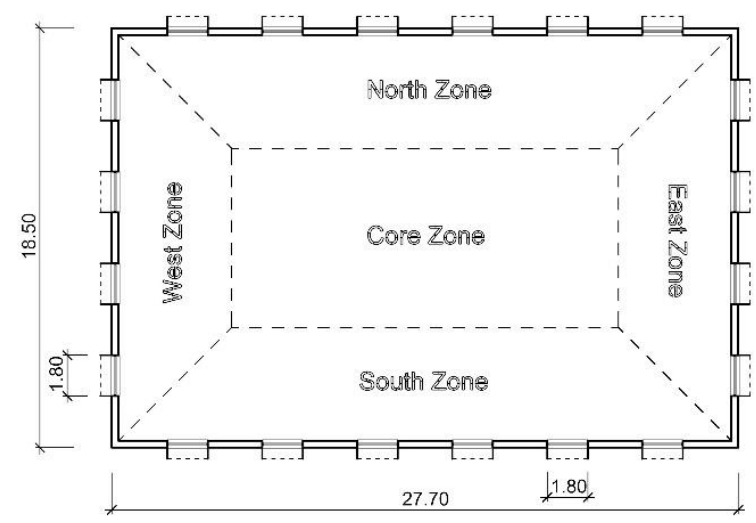

Figure 3: Building model (plan view).

Twenty similarly sized $(1.8$ by $1.8 \mathrm{~m})$ windows are distributed around the perimeter (12 windows on the south and north facades and 8 windows on the east and west facades). Each window has a $0.5 \mathrm{~m}$ deep overhang and two lateral $0.5 \mathrm{~m}$ deep fins (Figure 4). The opaque part of the exterior walls is modelled in terms of two thermally distinct (inner and outer) layers. 


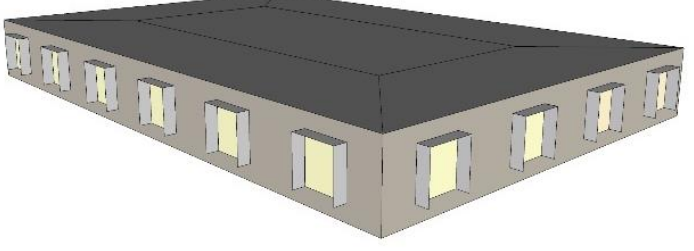

Figure 4: Building model (perspective).

The office is assumed to be occupied by a total of 31 inhabitants ( 9 in the core zone, 7 each in the south and north zones, and 4 each in the east and west zones). Daylighting control sensor points are assigned to perimeter zones (half-way on the middle axis between the front and back walls, $0.8 \mathrm{~m}$ above the floor). Each window is assumed to have a movable exterior blind. Natural ventilation at the perimeter zones is controlled based on the outside air temperature.

\section{Definition of the clusters}

In this example, the optimization variables have been categorized in terms of four clusters. Three clusters entail design parameters and one pertains to control variables.

\section{Facade geometry $(F G)$}

This cluster includes the dimension of the windows (d1) as well as the depth of overhangs (d2) and fins (d3) (Figure 5). For each façade, the dimensions of the windows and the depth of overhangs/fins vary simultaneously. As a whole, this cluster has 12 variables.

\section{Material Properties (MP)}

The façade is modelled in terms of opaque and transparent (glazing) components. The opaque part consists of an outer layer with thermal conductivity and density as pertinent variables. The relevant variables of the glazing are assumed to be the thermal transmittance (U-value) and the visible transmittance. Note that, for the purposes of the present demonstration, the glazing g-value is obtained as a function of its visible transmittance. Overall, this cluster has four variables.

\section{Blind (movable shading device) Properties (BP)}

This cluster comprises the pertinent variables of the movable blind, namely Slat Width, Slat Angle, and Slat Solar Reflectance (Figure 6). Slat separation is obtained as a function of the slat width. Given the independence of the four facades in view of blind operation, this cluster entails 12 variables.

\section{Control (CO)}

The final cluster is concerned with operation of blinds and windows. For blinds, the variable subjected to optimization is a threshold value for incident solar irradiance, above which the blinds are closed. Regarding ventilation, a base mechanical ventilation system is assumed that delivers a prescribed fresh air supply rate of 7 1.s-1 per person. However, given appropriate conditions (expressed in terms of an outdoor ambient temperature band), an additional magnitude of fresh air flow via window operation is supplied. In this case, the variables subject to optimization are threshold outdoor temperature values above (or below) which the windows are to be kept closed. This cluster includes thus 12 variables ( 4 for blind and 8 for window operation).

Table 1 shows an overview of the clusters and variables.

\section{Implementation platform}

A Java-based platform was developed to integrate the simulation and optimization tools and to automate the procedure. The energy analysis and thermal load simulation program EnergyPlus was coupled with GenOpt (Wetter, 2016). In this case, the optimization algorithm GPSPSOCCHJ was used (a Hybrid Generalized Pattern Search Algorithm with Particle Swarm Optimization Algorithm for Continuous and Discrete Variables).

\section{Objective function}

The selected performance indicators in the present experiment (and the basis for the definition of the cost function $(\mathrm{U})$ ) include the building's annual heating $(\mathrm{H})$, cooling (C), and lighting (L) energy demands. To establish the cost function, it is assumed that the building's heating system uses natural gas and has an $85 \%$ efficiency. The cooling system was assumed to be electrically driven with a COP of 3.5. Furthermore, it was assumed that, for the same energy content, the electricity price is 1.8 times the price of natural gas. This results in the following simple formulation for the cost function (U) (Equation 1), whereby $\mathrm{H}, \mathrm{C}$, and L (as well as $\mathrm{U}$ ) are in units of $\mathrm{kWh} \cdot \mathrm{m}^{-2}$.

$$
U=\left(\frac{H}{0.85}\right)+\left(\frac{C}{3.5} \times 1.8\right)+L \times 1.8
$$

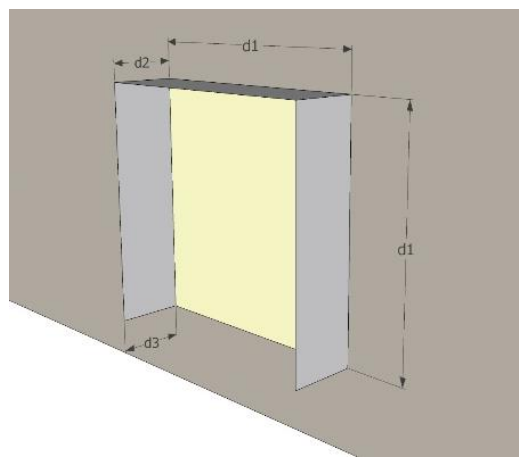

Figure 5: Variables of the cluster Façade Geometry (FG).

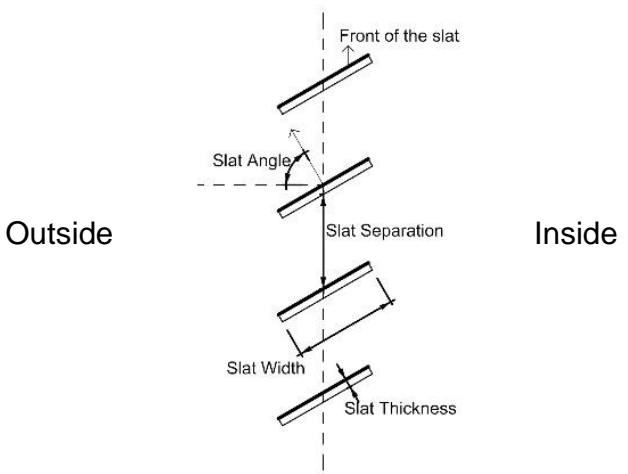

Figure 6: Blind properties (specified as per EnergyPlus input instruction). 
Table 1: Summary table of the clusters and variables.

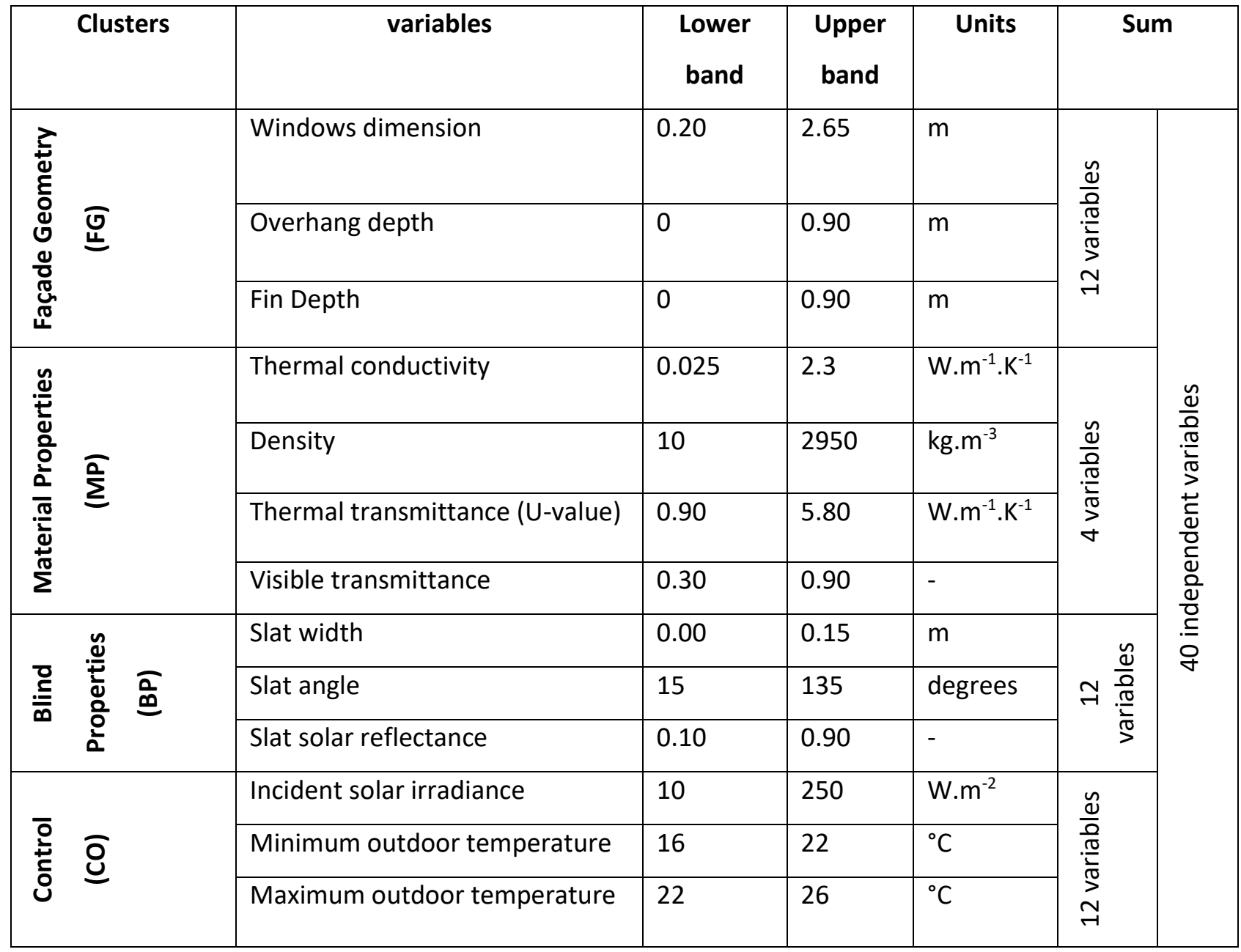

\section{The iteration logic}

In this example, a random mode of cycling between attribute clusters was selected (Figure 7).

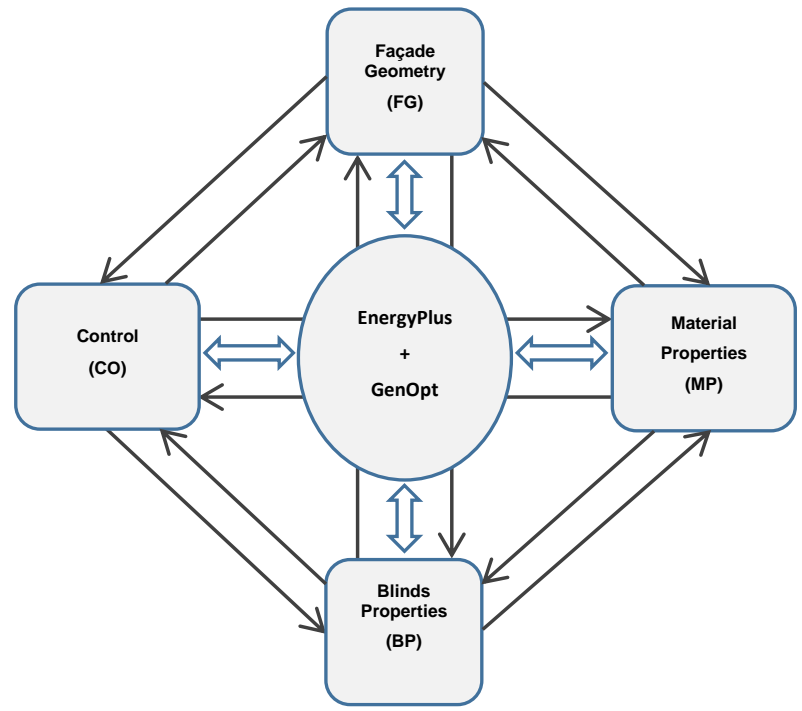

Figure 7: Illustration of random cycling between the attribute clusters.

\section{Results}

Figures 8 to 10 illustrate the main results of this case study. Thereby, abbreviations BM and GO refer to "Base Model" and "Global Optimization" respectively. Figure 8 illustrates the evolution of the cost function together with the total energy demand during 12 random iterations amongst attribute clusters. Figure 9 shows the energy demand (heating, lighting, cooling, and total) evolution for the same iterations. Figure 10 provides further details regarding the energy demand at the zone level.

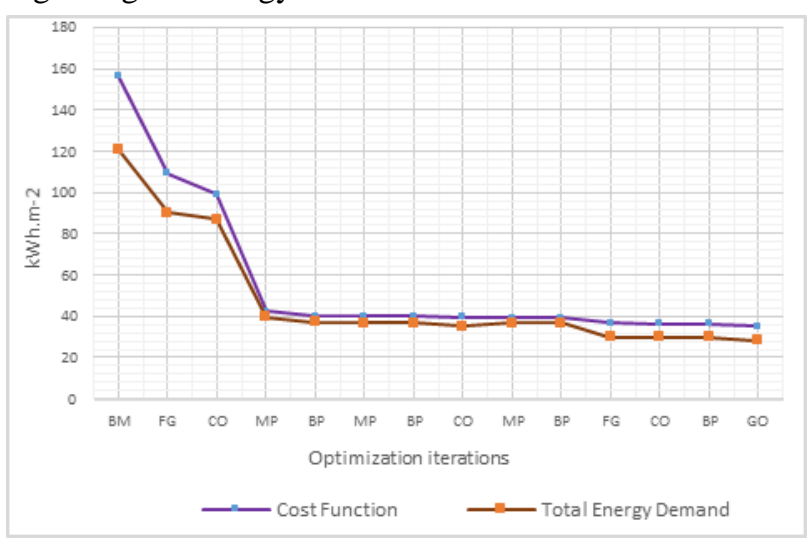

Figure 8: Cost function versus total energy demand. 


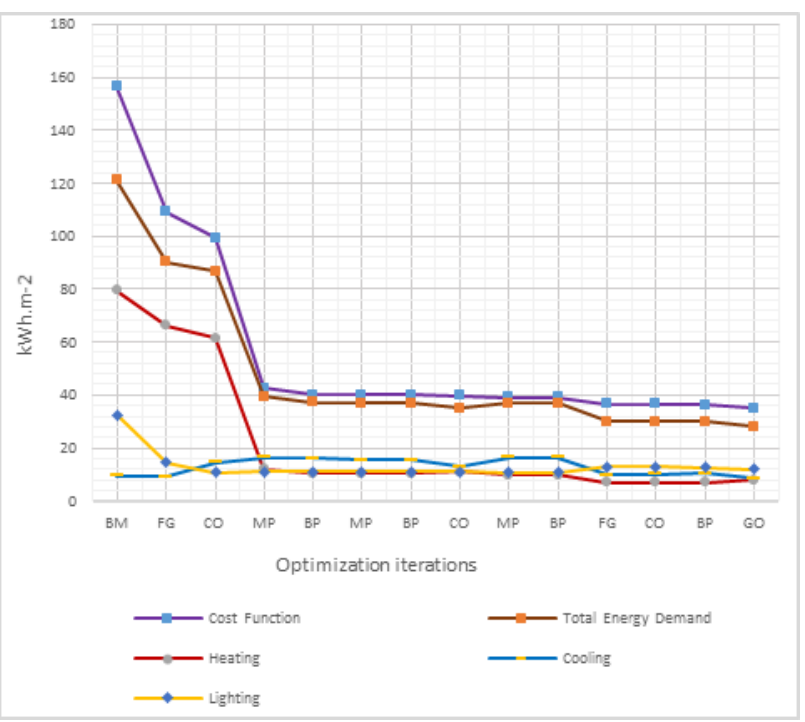

Figure 9: Energy demand evolution.

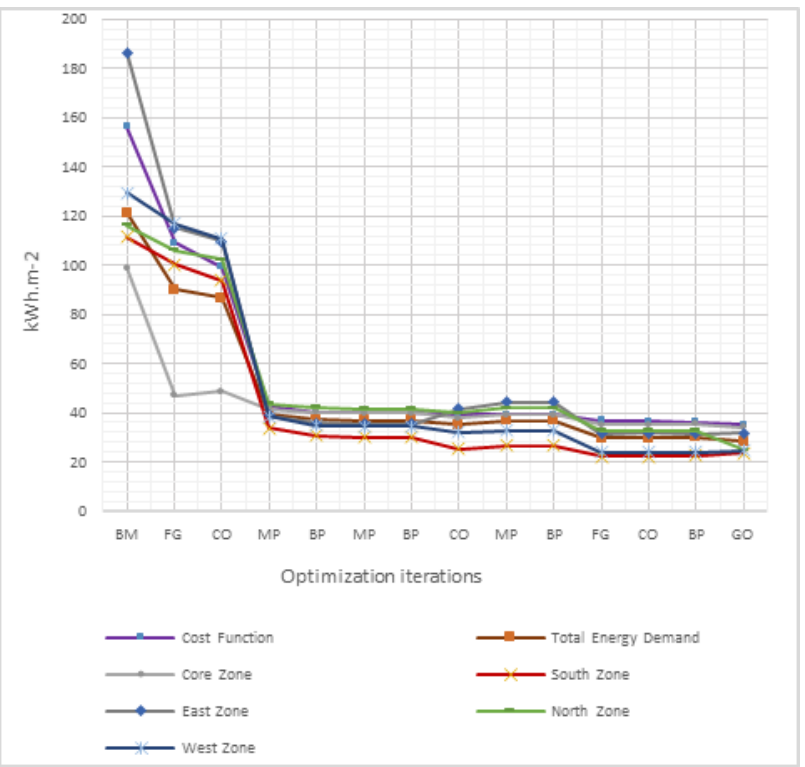

Figure 10: Energy demand evolution in each zone.

\section{The second illustrative case study}

\section{Building and energy model}

The base model of the second case study is a 20 floors tower office building in Vienna (Figure 11). Each $4 \mathrm{~m}$ high floor is an open office modelled as a thermal zone. Therefore, the model has 20 thermal zones. Each floor has a four-sided polygon shape. The radius of the peripheral circle of the first-floor polygon (the distance from the centre to the outer edge) is $5 \mathrm{~m}$. The scale of the floors changes in a sinusoid pattern from the bottom to the top of the tower. Each floor rotates $5^{\circ}$ with reference to the floor below. There is a pipe-wire skin at a distance of $1 \mathrm{~m}$ from the building's envelope. $50 \%$ of each façade consists of glazing. Half of the glazing area is operable on each side. There are two horizontal shading elements on each window. Each shading element is $0.5 \mathrm{~m}$ deep and $5 \mathrm{~cm}$ thick with a $45^{\circ}$ inclination.

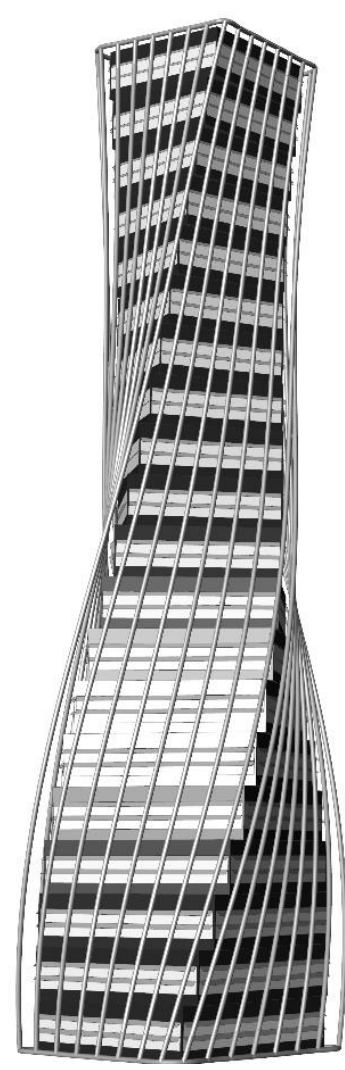

Figure 11: Perspective view of the tower.

The reflectance and emissivity of the shading surface are $50 \%$ and $80 \%$ respectively. Constructions of the opaque and glazing parts were retrieved from the construction library of EnergyPlus based on the ASHRAE climate zone of Vienna. Natural ventilation of each zone is controlled based on the outside air temperature. The shading elements are controlled based on the intensity of the solar radiation incident on the windows.

\section{Definition of the clusters}

\section{Building Geometry (BG)}

This cluster includes the geometry of the plan of the tower the number of the edges of the polygon can vary from 3 to 6 . The rotation of the successive floors can change between $0^{\circ}$ to $10^{\circ}$.

The façade's window to wall ratio represents a further variable with values that can vary between $10 \%$ and $90 \%$. The length of the shading slat varies from 0 to $0.6 \mathrm{~m}$. The number of the shading elements on each window can be between 0 and 5 . The horizontal or vertical position of the shadings is also a variable. Overall, this cluster has 6 independent variables.

\section{Material properties and Constructions (MC)}

The reflectance and emissivity of the shading (varying between $5 \%$ to $95 \%$ ), external walls, roof and window constructions represent the 5 variables of this cluster. All variables are discrete and have been extracted from the construction libraries of EnergyPlus.

\section{Control (CO)}

The last cluster is concerned with operation of windows and shades during the year. For shades, the variables 
subjected to optimization are the threshold value for incident solar irradiance, above which the shades are deployed on, and the angle of the shading slats. The ventilation control strategy is identical with the one in the first case study. This cluster includes thus 4 variables.

Table 2 shows an overview of the clusters and variables.

\section{Implementation platform}

A platform developed in Rhino/Grasshopper. Ladybug and Honeybee were used for energy simulation. Ladybug tools is a collection of free computer applications that support environmental design and education. Ladybug Tools connects 3D Computer-Aided Design (CAD) interfaces to a host of simulation engines (Sadeghipour et al. 2018). Honeybee supports detailed daylighting and thermodynamic modelling. Specifically, it creates, runs, and visualizes the results of energy models using EnergyPlus/OpenStudio. It accomplishes this by linking the simulation engines to Rhino/Grasshopper as CAD and visual scripting interfaces.

The selected optimizer for the implementation was Opossum, a recently introduced optimization plug-in for Grasshopper. It is a publicly available, model-based optimization tool aimed at architectural design optimization and especially applicable to problems that involve time-intensive simulations regarding, for instance, daylighting and energy use (Wortmann, 2017).

\section{Objective function}

The nature of the selected objective function for this second case study is identical with the aforementioned first case study (Equation 1).

\section{The iteration logic}

The approach of optimization iterations is a predefined cycling between attribute clusters (Figure 12).

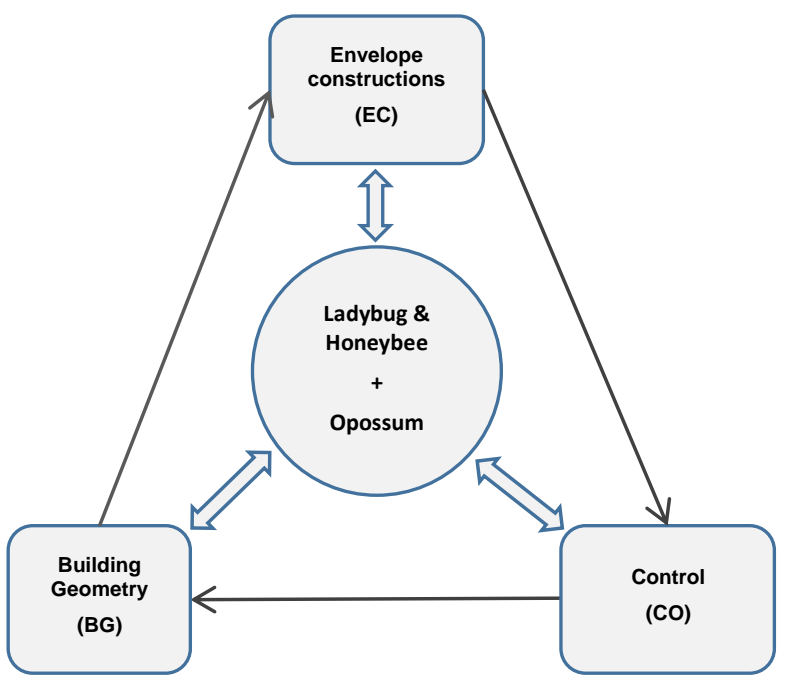

Figure 12: Schematic illustration of the platform and iterations between the attribute clusters.

Table 2: Summary table of the clusters and variables.

\begin{tabular}{|c|c|c|c|c|c|c|}
\hline Clusters & Variables & Lower bound & Upper bound & Units & \multicolumn{2}{|c|}{ Sum } \\
\hline \multirow{6}{*}{ 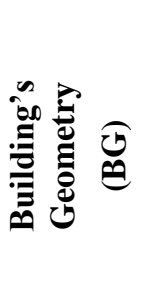 } & Number of polygon edges & 3 & 6 & - & \multirow{6}{*}{ 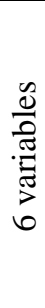 } & \multirow{15}{*}{ 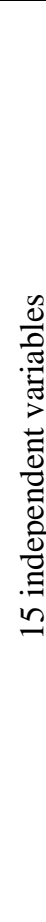 } \\
\hline & rotation of the successive floors & 0 & 10 & $\circ$ & & \\
\hline & Window to wall ratio & 10 & 90 & $\%$ & & \\
\hline & Shading device position $*$ & - & - & - & & \\
\hline & Shading device depth & 0 & 0.60 & $\mathrm{~m}$ & & \\
\hline & Number of shading devices & 0 & 5 & - & & \\
\hline \multirow{5}{*}{ 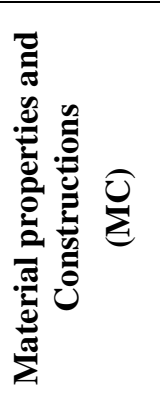 } & External walls $* *$ & - & - & - & \multirow{5}{*}{ 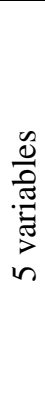 } & \\
\hline & Windows $* *$ & - & - & - & & \\
\hline & Roofs ** & - & - & - & & \\
\hline & Shading slat reflectance & 5 & 95 & $\%$ & & \\
\hline & Shading slat emissivity & 5 & 95 & $\%$ & & \\
\hline \multirow{4}{*}{ 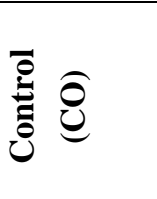 } & Incident solar irradiance & 10 & 150 & $\mathrm{~W} / \mathrm{m}^{2}$ & \multirow{4}{*}{ 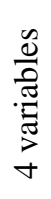 } & \\
\hline & Shading device angle & 0 & 90 & $\circ$ & & \\
\hline & Minimum outdoor temperature & 18 & 22 & ${ }^{\circ} \mathrm{C}$ & & \\
\hline & Maximum outdoor temperature & 22 & 26 & ${ }^{\circ} \mathrm{C}$ & & \\
\hline
\end{tabular}

*This is a Boolean variable and can be horizontal or vertical.

**These variables are discrete and been derived from EnergyPlus constructions library (accessible through Honeybee tools). 


\section{Results}

The results of the second illustrative case study are shown in Figures 13 and 14. Figure 13 illustrates the evolution of the cost function together with the total energy demand in the course of 6 iterations between attribute clusters. Figure 14 shows the energy demand (heating, lighting, cooling, and total) evolution for the same iterations.

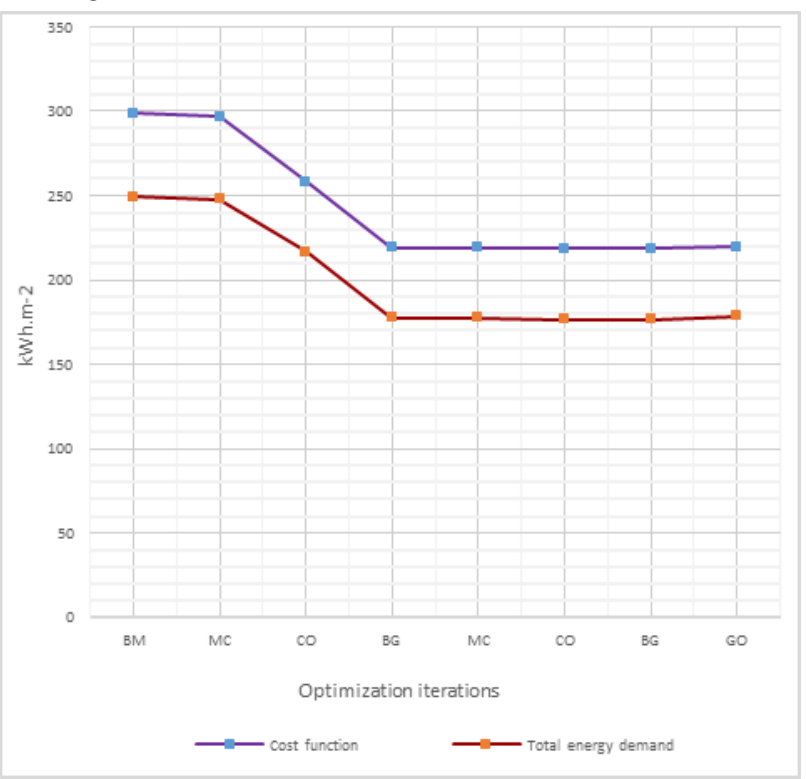

Figure 13: Cost function versus total energy demand.

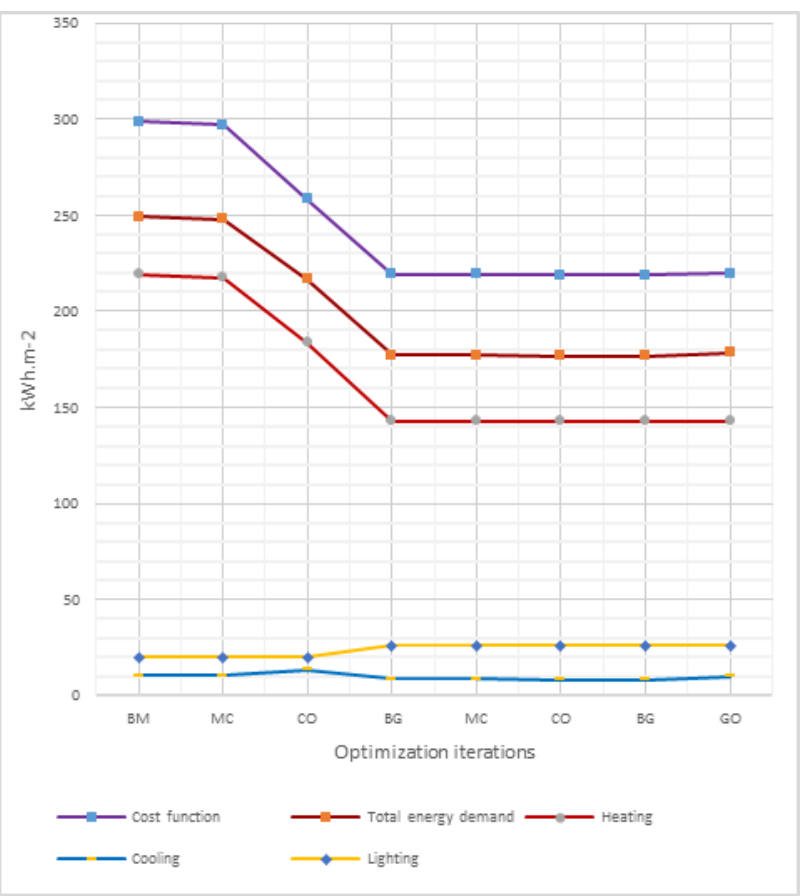

Figure 14: Detailed annual energy demand normalized by area as well as the cost function.

\section{Discussion}

Both presented examples display the rapid convergence toward optima, as indicated by decrease in value of energy use indicators and the cost functions (see Figures 8, 9, 10, 13, and 14). The bulk of optimization-based design improvement is in fact achieved during the first three to five iterations.

As such, the end values of the individual design variables are not necessarily identical with those in the global optimization scenario. Interestingly, the proposed intra-cluster cycling approach in both case studies delivers better results with regard to the time and cost function. Specifically speaking, the global optimization requires roughly twice as much computational time as the proposed intra-cluster cycling approach.

\section{Conclusion}

We reported on the progress regarding a novel approach toward iterative global optimization of locally optimized attribute clusters of building design solutions. If wellstructured, such clusters of design space attributes can be easily comprehensible to typical building designers as a compound yet coherent aspects of a design (e.g., building enclosure, building materials, building geometry, building systems, control strategies). Thus, grouped clusters can be made subject to multiple passes of local simulation-assisted optimizations instead of a single-pass black-box global optimization step. We provided a proof of concept of the proposed approach via a prototypical implementation using existing simulation and optimization tools.

While not explicitly covered in this contribution, the implementation provides the users with degrees of freedom in view of the selection of the clusters to be optimized. Moreover, additional clusters can be defined and variable sets in each cluster can be manipulated, while still achieving convergence within reasonable temporal horizons.

We illustrated the performance of the current implementation of the proposed approach via two optimization case studies, which contained different complexities (i.e., a simple rectangular one-story building and a tower with more complicated geometry) as well as different iteration approach (random and predefined cycling between the attribute clusters)

The results thus far are highly encouraging: The proposed method delivers optimized solutions (both at building and zone levels) that are - as far as the values of the energy performance indicators and the associate cost functions are concerned - virtually indistinguishable from those of reference one-shot global optimization runs. However, in our approach, the results are not only obtained more efficiently, but also via a process that is arguably more transparent, traceable, and designer-friendly. 


\section{References}

Fiske, V. P., \& Bhonde, C. B. (2015). Energy Simulation in Buildings. International Journal of Scientific \& Engineering Research 6, 137-143.

Mahdavi, A., Shirdel, H., \& Tahmasebi, F. (2016). A novel approach to building performance optimization via iterative operations on attribute clusters of design options. Proceedings from ECPPM2016: $11^{\text {th }}$ European Conference on Product and Process Modelling. Limassol (Cyprus).

Nguyen, A., Reiter, S., Rigo, P. (2014). A review on simulation-based optimization methods applied to building performance analysis. Applied Energy 113, 1043-1058.

Sadeghipour Roudsari, M., \& Mackey, C. (2018). Ladybug tools: http://www.ladybug.tools/.

Shirdel, H., Tahmasebi, F., \& Mahdavi, A. (2018). Convergence toward optimal building designs via multiple iterative local optimization applied to attribute clusters of design variants. Proceedings of BauSIM2018. Karlsruhe (Germany).

Wetter, M. (2016). GenOpt, Generic Optimization Retrieved from: https://Simulationresearch.lbl.gov/

Wortmann, T. (2017). Opossum: Introducing and Evaluating a Model-based Optimization Tool for Grasshopper. Proceeding from CAADRIA2017: 22 ${ }^{\text {nd }}$ International Conference of the Association for Computer-Aided Architectural Design Research in Asia. Hong Kong (China).

Yang, C., Li, H., Rezgui, Y., Petri, I., Yuce, B., Chen, B., \& Jayan, B. (2014). High throughput computing based distributed genetic algorithm for building energy consumption optimization. Energy and Buildings 76, 92-101. 